\title{
A Continuous Max-Flow Approach to Potts Model
}

\author{
Jing Yuan ${ }^{1}$, Egil Bae ${ }^{2}$, Xue-Cheng Tai ${ }^{2,3}$, and Yuri Boykov ${ }^{1}$ \\ 1 Computer Science Department, University of Western Ontario, London Ontario, \\ Canada N6A 5B7 ((cn.yuanjing, yboykov)@gmail.com). \\ 2 Department of Mathematics, University of Bergen, Norway ((Egil.Bae, \\ tai)@math.uib.no). \\ 3 Division of Mathematical Sciences, School of Physical and Mathematical Sciences, \\ Nanyang Technological University, Singapore. (tai@mi.uib.no).
}

\begin{abstract}
We address the continuous problem of assigning multiple (unordered) labels with the minimum perimeter. The corresponding discrete Potts model is typically addressed with a-expansion which can generate metrication artifacts. Existing convex continuous formulations of the Potts model use TV-based functionals directly encoding perimeter costs. Such formulations are analogous to 'min-cut' problems on graphs. We propose a novel convex formulation with a continous 'max-flow' functional. This approach is dual to the standard TV-based formulations of the Potts model. Our continous max-flow approach has significant numerical advantages; it avoids extra computational load in enforcing the simplex constraints and naturally allows parallel computations over different labels. Numerical experiments show competitive performance in terms of quality and significantly reduced number of iterations compared to the previous state of the art convex methods for the continuous Potts model.
\end{abstract}

\section{Introduction}

The multi-partitioning problem, or multi-labeling problem, was extensively investigated in image processing and computer vision [1]. It computes the optimal labeling $l \in l_{1}, \ldots, l_{n}$ of each graph node or image pixel. Looking for such optimal labeling function with respect to some energy functional is an important mathematical strategy to model a wide range of applications, e.g. image segmentation $[2,3], 3 \mathrm{D}$ reconstruction [4] etc. In this work, we focus on the Potts model that does not favor any particular order of the labels. The Potts model is also referred to as a piecewise constant labeling model which minimizes the total perimeter of the one-label (constant) regions.

In a discrete setting, Potts model corresponds to a practically important special case of a Markov Random Field (MRF) defined over a graph [5]. A typical MRF energy sums unary potentials defined over graph nodes and pairwise potentials defined over graph edges. When pixels can take only one of 2 labels, the resulting binary energy function can be efficiently and globally minimized by 
graph cuts [6], provided that the pairwise potentials are submodular [7]. However, for more than two labels typical MRF optimization problems are NP hard, so is Potts model. In particular, Potts model corresponds to a multi-terminal graph cut problem where only provably good approximate solutions are guaranteed, for example, via $\alpha$-expansion or $\alpha-\beta$ swap [2] and some LP relaxations $[8,9]$. Another drawback of the discrete setting is that the results are often biased by the discrete grid causing metrication errors. Such visual artifacts can be largely reduced by either adding more neighbour nodes $[10,11]$ or applying high-order clique [12]. However, extra computation and memory load are introduced.

Parallel to these developments, variational methods have been proposed for solving the same Potts model in the spatially continuous setting where a bounded image domain is considered. In this regard, level set introduces the most direct and natural way to encode the piecewise constant labeling function and its related computation provides an efficient way to resolve the optimal partitions with a subgrid accuracy, see e.g. [13-15] and its variant of the piecewise constant level set method (PCLSM) $[16,17]$. Unfortunately, these formulations are nonconvex and computation often gets stuck in a local minima. Recently, convex relaxation approaches were proposed, e.g. [3, 18-22]. Comparing to level set methods, Great advantages in numerics can be achieved, e.g. reliable algorithms can be build up by standard convex optimization theories [23]. Since a strict mathematical proof of the exactness of such a convex relaxation approach to the nonconvex Potts model is still open and argued, its approximation result can only be accepted as suboptimal. One may claim the convex relaxation method gives the solution which is closer to the exact global minimum than the local minima by the level set formulation. Our experiment results confirmed this.

In this paper, we study and solve the Potts problem in the spatially continuous setting through its convex relaxed formulation, i.e. the convex relaxed Potts model. In $[18,22]$, such convex minimization problem is computed directly through the minimization over the labeling functions, i.e. tackle the minimal cut problem in a direct way, extra computation load is introduced to explore the pointwise simplex constraint within each iteration. Bae et. al. [21] proposed an equivalent dual model and its associated smoothing formulation based on the maximum entropy regularization, which properly avoids the extra step to handle simplex constraints and leads to a much simpler numerical scheme. To the best of our knowledge, none of previous works investigates the potential max-flow formulation which is dual to the concerning minimal cut. This is in contrast to the discrete case, where the minimal cut of a graph is often studied and computed over its dual maximal flow formulation, most efficient algorithms of graph-cuts were designed and explained in a flow maximization manner [24]. We devote this work to study the max-flow model associated to the convex relaxed Potts model. We also propose a fast max-flow based algorithm for computing continuous mincuts. Experiments show that our max-flow algorithm is much more efficient than the state of art of computational methods [18,22]. 
Contributions We summarize our main contributions in this paper as follows: first, we propose the novel max-flow formulation to the minimal cut of the given continuous image domain, i.e. the convex relaxed Potts problem. We show the studied max-flow and min-cut models are equivalent and dual to each other, hence the convex relaxed Potts problem can be solved through the proposed max-flow formulation. Analysis of the max-flow problem also leads to a new variational perspective of the corresponding minimal cut or continuous Potts problem. In addition, we build up the new multiplier-based max-flow algorithm upon the equivalent primal-dual model. It is numerically reliable and efficient. Its convergence can be proved by classical optimization theories. Our experiments show it is around 4 times faster than the previous methods [18,22]. Last but not least, such algorithm has a natural parallel framework over labeling functions and can, therefore, be easily implemented and accelerated on a parallel platform.

\section{Convex Relaxed Potts Model and Previous Works}

\subsection{Convex Relaxed Potts Model}

The Potts model originates from the statistical physics [25] and its spatially continuous version tries to partition the continuous domain $\Omega$ into $n$ disjoint subdomains $\left\{\Omega_{i}\right\}_{i=1}^{n}$ by

$$
\begin{array}{ll}
\min _{\left\{\Omega_{i}\right\}_{i=1}^{n}} & \sum_{i=1}^{n} \int_{\Omega_{i}} \rho\left(l_{i}, x\right) d x+\lambda \sum_{i=1}^{n}\left|\partial \Omega_{i}\right| \\
\text { s.t. } & \cup_{i=1}^{n} \Omega_{i}=\Omega, \quad \Omega_{k} \cap \Omega_{l}=\emptyset, \forall k \neq l
\end{array}
$$

where $\left|\partial \Omega_{i}\right|$ measures the perimeter of each disjoint subdomain $\Omega_{i}, i=1 \ldots n$. The function $\rho\left(l_{i}, x\right), i=1 \ldots n$, evaluates the performance of assigning the label $l_{i}$ to the specified position $x$. As a special case, the piecewise constant MumfordShah functional can be encoded in terms of (1) with $\rho\left(l_{i}, x\right)=\left|I(x)-l_{i}\right|^{p}$ where $l_{1} \ldots l_{n}$ are the given grayvalue constants. Obviously, Potts model favors the labeling with 'tight' boundaries.

Let $u_{i}(x), i=1 \ldots n$, denote the indicator function of the disjoint subdomain $\Omega_{i}$, i.e.

$$
u_{i}(x):=\left\{\begin{array}{ll}
1, & x \in \Omega_{i} \\
0, & x \notin \Omega_{i}
\end{array}, \quad i=1 \ldots n .\right.
$$

The perimeter of each disjoint subdomain can be computed by

$$
\left|\partial \Omega_{i}\right|=\int_{\Omega}\left|\nabla u_{i}\right| d x, \quad i=1 \ldots n .
$$

The Potts model (1) can then be rewritten as

$$
\min _{u_{i}(x) \in\{0,1\}} \sum_{i=1}^{n} \int_{\Omega}\left\{u_{i}(x) \rho\left(l_{i}, x\right)+\lambda\left|\nabla u_{i}\right|\right\} d x, \text { s.t. } \sum_{i=1}^{n} u_{i}(x)=1, \forall x \in \Omega
$$


where the constraints to $u_{i}(x), i=1 \ldots n$, just corresponds to the condition (2) of subdomains $\Omega_{i}, i=1 \ldots n$.

Clearly, the Potts model (4) is nonconvex due to the binary configuration of each function $u_{i}(x) \in\{0,1\}$. The convex relaxed Potts model [20,22, 21] proposes to relax such binary constraints to the convex interval $[0,1]$ and approximates (4) by the reduced convex optimization problem:

$$
\min _{u \in S} \sum_{i=1}^{n} \int_{\Omega} u_{i}(x) \rho\left(l_{i}, x\right) d x+\alpha \sum_{i=1}^{n} \int_{\Omega}\left|\nabla u_{i}\right| d x
$$

where $S$ is the convex constrained set of $u(x):=\left(u_{1}(x), \ldots, u_{n}(x)\right)$ :

$$
S=\left\{u(x) \mid\left(u_{1}(x), \ldots, u_{n}(x)\right) \in \triangle_{+}, \forall x \in \Omega\right\},
$$

$\triangle_{+}$is the simplex set, i.e.

$$
\text { for } \forall x \in \Omega, \quad \sum_{i=1}^{n} u_{i}(x)=1 ; \quad u_{i}(x) \in[0,1], \quad i=1 \ldots n .
$$

The computation result of the convex relaxed Potts model (5) gives rise to a cut of the continuous image domain $\Omega$ with multiple terminals. (5) is, therefore, also called the continuous min-cut model in this paper. This is in comparison to its equivalent max-flow formulation proposed in later sections.

\subsection{Previous Works}

In [18], Zach et al introduced an alternating optimization approach to solve (5) in a numerically splitting way:

$$
\min _{u, v \in S} \sum_{i=1}^{n} \int_{\Omega} v_{i}(x) \rho\left(l_{i}, x\right) d x+\frac{1}{2 \theta}\|u-v\|^{2}+\alpha \sum_{i=1}^{n} \int_{\Omega}\left|\nabla u_{i}\right| d x .
$$

Obviously, when $\theta$ takes a value small enough, the above convex optimization problem properly approximates the convex relaxed Potts model (5). Within each iteration, two substeps are taken to tackle the total-variation term and explore the pointwise simplex constraint $S$ respectively.

In [22], a Douglas-Rachford splitting algorithm was proposed to solve a quite similar problem as (5), where a variant of the total-variation term is considered:

$$
\int_{\Omega} \sqrt{\left|\nabla u_{1}(x)\right|^{2}+\ldots+\left|\nabla u_{n}(x)\right|^{2}} d x .
$$

As in [18], the proposed splitting procedure involves an outer loop with two substeps, where the first substep solves a tv minimization problem iteratively until convergence, while the second substep projects the current solution to the convex set $S$. 
In $[20,26]$, the authors introduced another relaxation based on a multi-layered configuration, which was shown to be tighter. A more complex constraint on the dual variable $p$ is given to avoid multiple countings. In addition, a PDE-based projection-descent scheme was applied to achieve the minimum.

In contrast to $[18,22,20,26],[21]$ did not try to tackle the labeling function of the continuous min-cut problem (5) directly, but solved its equivalent dual formulation:

$$
\max _{p_{i} \in C_{\alpha}} \int_{\Omega}\left\{\min \left(\rho\left(l_{1}, x\right)+\operatorname{div} p_{1} \ldots \rho\left(l_{n}, x\right)+\operatorname{div} p_{n}\right)\right\} d x .
$$

where $\operatorname{div} p_{i}, i=1 \ldots n$, correspond to the total-variation terms under the dual perspective and the convex set $C_{\alpha}$ is defined as

$$
C_{\alpha}=\left\{p\left|\|p\|_{\infty} \leq \alpha, p_{n}\right|_{\partial \Omega}=0\right\} .
$$

Once the optimal functions $p_{i}^{*}(x), i=1 \ldots n$, were resolved, the labeling functions $u_{i}(x), i=1 \ldots n$, can be simply recovered by

$$
u_{k}^{*}(x)=\left\{\begin{array}{ll}
1 & \text { if } k=\arg \min _{i=1 \ldots n} \rho\left(l_{i}, x\right)+\operatorname{div} p_{i}^{*}(x) \\
0 & \text { otherwise }
\end{array} .\right.
$$

provided the above argmin is unique. It was further shown by [21] that the highly nonsmooth dual formulation (6) can be properly approximated by the maximization of a smooth energy function, i.e.

$$
\max _{p_{i} \in C_{\lambda}}-s \int_{\Omega}\left\{\log \sum_{i=1}^{n} \exp \left(\frac{-f_{i}-\operatorname{div} p_{i}}{s}\right)\right\} d x .
$$

Such a smooth dual model (9) approaches (6) with a maximum entropy regularizer and can be solved efficiently by a simple and reliable algorithmic scheme due to its smoothness and convexity.

In this paper, we propose a new continuous max-flow formulation which is equivalent to the continuous min-cut model (5), actually dual to each other. In theory, it provides a new variational perspective to investigate the continuous min-cut with multiple terminals or labels. In numerics, its great advantages over previous works are: it avoids pointwise projections onto the simplex constraint $S$ within each outer loop as [18,22]; in comparison to [21], it exactly solves (6) without any smoothing procedure; it is globally optimized based on an efficient and reliable multiplier-based max-flow algorithm, in contrast to the PDE-descent method $[20,26]$ whose convergence may suffer from uncareful stepsizes resulting in suboptimums; experiments show a faster convergence rate, about 4 times, than $[18,22]$.

\section{Continuous Max-Flow Model}

In this section, we introduce the novel continuous max-flow formulation to the continuous min-cut problem (5) with $n$ labels. 


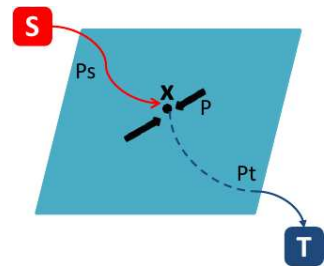

(a)

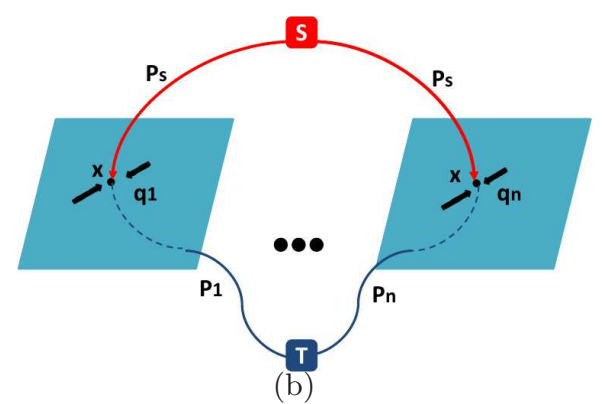

Fig. 1. (a) Continuous settings of max-flow with two labels; (b) Continuous configuration of max-flow with $n$ labels.

\subsection{Continuous Max-Flow Model}

Continuous Max-Flow Model with 2 Labels Before we introduce the continuous max-flow model with $n$ labels, we first introduce the recent study of the continuous max-flow model with 2 labels proposed by the authors [27] which is dual to the continuous $s$ - $t$ cut. This is directly analoguous to the graph-based max-flow and $s$ - $t$ cut: given the continuous image domain $\Omega$, we assume there are two terminals, the source $s$ and the sink $t$, see figure (a) of Fig. 1. We assume that for each image position $x \in \Omega$, there are three concerning flows: the source flow $p_{s}(x) \in \mathbb{R}$ directed from the source $s$ to $x$, the sink flow $p_{t}(x) \in \mathbb{R}$ directed from $x$ to the sink $t$ and the spatial flow field $p(x) \in \mathbb{R}^{2}$. The three flow fields are constrained by capacities

$$
p_{s}(x) \leq C_{s}(x), \quad p_{t}(x) \leq C_{t}(x), \quad|p(x)| \leq C(x) ; \quad \forall x \in \Omega .
$$

In addition, for $\forall x \in \Omega$, all flows are conserved, i.e.

$$
p_{t}-p_{s}+\operatorname{div} p=0, \quad \forall x \in \Omega .
$$

Therefore, we formulate the corresponding max-flow problem by maximizing the total flow from the source:

$$
\max _{p_{s}, p_{t}, p} \int_{\Omega} p_{s} d x
$$

subject to flow constraints (10) and (11).

Yuan et al [27] proved that such a continuous max-flow formulation (12) is equivalent to the continuous $s$ - $t$ min-cut problem $[3,28]$ as follows:

$$
\min _{u(x) \in[0,1]} \int_{\Omega}(1-u) C_{s} d x+\int_{\Omega} u C_{t} d x+\int_{\Omega} C(x)|\nabla u| d x .
$$

Actually, (13) just gives the dual model to (12) and the labeling function $u(x)$ is the multiplier to the flow conservation condition (11). Furthermore, an efficient and reliable max-flow based algorithm can be built up through (12). 
Continuous Max-Flow Model with $\mathbf{n}$ Labels Motivated by the above observations, we give a continuous configuration of the max-flow model with $\mathrm{n}$ labels, see figure (b) of Fig. 1:

1. $n$ copies $\Omega_{i}, i=1 \ldots n$, of the image domain $\Omega$ are given in parallel;

2. For each position $x \in \Omega$, the source flow $p_{s}(x)$ tries to stream from the source $s$ to $x$ at each copy $\Omega_{i}, i=1 \ldots n$, of $\Omega$. The source flow field is the same for each $\Omega_{i}, i=1 \ldots n$, i.e. $p_{s}(x)$ is unique;

3. For each position $x \in \Omega$, the sink flow $p_{i}(x), i=1 \ldots n$, is directed from $x$ at the $i$-th copy $\Omega_{i}$ to the sink $t$. The $n$ sink flow fields $p_{i}(x), i=1 \ldots n$, may be different;

4. The spatial flow fields $q_{i}(x), i=1 \ldots n$, are defined within each copy $\Omega_{i}$, $i=1 \ldots n$. They may also be different from each other.

For such a contiuous setting, we give the constrained conditions for flows $p_{i}(x)$ and $q_{i}(x)$, at $x \in \Omega$, as follows

$$
\begin{gathered}
\left|q_{i}(x)\right| \leq C_{i}(x), \quad p_{i}(x) \leq \rho\left(\ell_{i}, x\right), \quad i=1 \ldots n \\
\left(\operatorname{div} q_{i}-p_{s}+p_{i}\right)(x)=0, \quad i=1, \ldots, n .
\end{gathered}
$$

Note: there is no constraint for the source flow $p_{s}(x)$.

We, then, formulate the respective continuous max-flow model, over all the flow fields $p_{s}(x), p(x):=\left(p_{1}(x), \ldots, p_{n}(x)\right)$ and $q(x):=\left(q_{1}(x), \ldots, q_{n}(x)\right)$, as

$$
\max _{p_{s}, p, q}\left\{P\left(p_{s}, p, q\right):=\int_{\Omega} p_{s} d x\right\}
$$

subject to (14) and (15).

In the following section, we introduce the equivalent models of the continuous max-flow formulation (16). We show its equivalent dual model just gives the continuous min-cut model (5) provided $C(x)=\alpha$.

Comments It is easy to notice that when the source flow $p_{s}(x)$ tries to pass the same position $x$ at each $\Omega_{i}, i=1 \ldots n$, in view of the flow conservation condition (15), we have

$$
p_{s}(x)=\operatorname{div} q_{i}(x)+p_{i}(x), \quad i=1 \ldots n .
$$

Observe the righthand of the above formulation and the configuration shown in Fig. $1, p_{s}(x)$ is constrained and should be given within a feasible set, i.e. consistent to all $n$ flow configurations of $\operatorname{div} q_{i}(x)+p_{i}(x), i=1 \ldots n$, at $x$. Consider the flow capacity constraint of $p_{i}(x)(14)$, it is easy to conclude that

$$
p_{s}(x)=\min \left(\operatorname{div} q_{1}(x)+\rho\left(l_{1}, x\right), \ldots, \operatorname{div} q_{n}(x)+\rho\left(l_{n}, x\right)\right), \quad \forall x \in \Omega .
$$

Therefore, the maximum of $\int_{\Omega} p_{s} d x$ suggests

$$
\max _{\left|q_{i}(x)\right| \leq C_{i}(x)} \int_{\Omega}\left\{\min \left(\rho\left(l_{1}, x\right)+\operatorname{div} q_{1}, \ldots, \rho\left(l_{n}, x\right)+\operatorname{div} q_{n}\right)\right\} d x,
$$


which discovers the dual model (6) of [21] when $C_{i}(x)=\alpha$ are constant.

We can consider each image copy $\Omega_{i}, i=1 \ldots n$, together with the constrained sink flow field $p_{i}(x)$ and the spatial flow field $q_{i}(x)$ given in (14), as a 'filter' $F_{i}$ whose capacity at $x \in \Omega$ is constrained by $\operatorname{div} q_{i}(x)+p_{i}(x)$. Then one can explain the max-flow model (16) such that all the filters $F_{i}, i=1, \ldots, n$, are layered one by one and the source flow $p_{s}(x)$ tries to pass such a stack of 'filters' in one time. It is obvious that $p_{s}(x)$ is bottlenecked by the minimum capacity of $\operatorname{div} q_{i}(x)+p_{i}(x), i=1 \ldots n$. In such a filter configuration, (16) aims to maximize the total flow passing this 'filter' set.

\subsection{Equivalent Primal-Dual Formulation}

We introduce the multiplier functions $u_{i}(x), i=1 \ldots n$, to the flow balance condition (15). Therefore, we have the equivalent primal-dual model of (16)

$$
\begin{aligned}
\max _{p_{s}, p, q} \min _{u}\left\{E\left(p_{s}, p, q ; u\right):=\int_{\Omega} p_{s} d x+\sum_{i=1}^{n} \int_{\Omega} u_{i}\left(\operatorname{div} q_{i}-p_{s}+p_{i}\right) d x\right\} \\
\text { s.t. } \quad p_{i}(x) \leq \rho\left(\ell_{i}, x\right), \quad\left|q_{i}(x)\right| \leq C_{i}(x) ; \quad i=1 \ldots n
\end{aligned}
$$

where $u(x):=\left(u_{1}(x), \ldots, u_{n}(x)\right)$.

Rearranging the energy function $E\left(p_{s}, p, q ; u\right)$ of (19), we have

$$
E\left(p_{s}, p, q ; u\right)=\int_{\Omega}\left\{\left(1-\sum_{i=1}^{n} u_{i}\right) p_{s}+\sum_{i=1}^{n} u_{i} p_{i}+\sum_{i=1}^{n} u_{i} \operatorname{div} q_{i}\right\} d x
$$

For the primal-dual model (19), the conditions of the minimax theorem (see e.g., [29] Chapter 6, Proposition 2.4) are all satisfied. That is, the constraints of flows are convex, and the energy function is linear in both the multiplier $u$ and the flow functions $p_{s}, p$ and $q$, hence convex l.s.c. for fixed $u$ and concave u.s.c. for fixed $p_{s}, p$ and $q$. This confirms the existence of at least one saddle point, see $[29,30]$. It also follows that the min and max operators of the primal-dual model (19) can be interchanged, i.e.

$$
\max _{p_{s}, p, q}\left\{\min _{u} E\left(p_{s}, p, q ; u\right)\right\}=\min _{u}\left\{\max _{p_{s}, p, q} E\left(p_{s}, p, q ; u\right)\right\} .
$$

\subsection{Equivalent Dual Formulation}

Now we investigate the optimization of (19) by the min-max order as the righthand side of (21), i.e. first maximize $E\left(p_{s}, p, q ; u\right)$ over the flow functions $p_{s}, p$ and $q$ then minimize over the multiplier function $u$. We show that this leads to the equivalent dual model of the continuous max-flow formulation (16), i.e.

$$
\begin{aligned}
\min _{u}\{D(u):= & \left.\sum_{i=1}^{n}\left(\int_{\Omega} u_{i}(x) \rho\left(\ell_{i}, x\right) d x+\int_{\Omega} C_{i}(x)\left|\nabla u_{i}\right| d x\right)\right\} \\
\text { s.t. } \quad & \sum_{i=1}^{n} u_{i}(x)=1, \quad u_{i}(x) \geq 0 .
\end{aligned}
$$


Optimization of Flow Functions $p, q$ and $p_{s}$ : In order to optimize the flow function $p(x)$ in (20), let us consider the following maximization problem

$$
f(q)=\max _{p \leq C} p \cdot q .
$$

where $p, q$ and $C$ are scalars. When $q<0, p$ can be chosen to be a negative infinity value in order to maximize the value $p \cdot q$, i.e. $f(q)=+\infty$. In consequence, we must have $q \geq 0$ so as to make the function $f(q)$ meaningful. Observe now that

$$
\left\{\begin{array}{l}
\text { if } q=0, \text { then } p \leq C \text { and } f(q) \text { reaches the maximum } 0 \\
\text { if } q>0, \text { then } p=C \text { and } f(q) \text { reaches the maximum } q \cdot C
\end{array} .\right.
$$

By virtue of (24), we can equally express $f(q)$ by

$$
f(q)=q \cdot C, \quad q \geq 0 .
$$

Apply (23) to the maximization of $E\left(p_{s}, p, q ; u\right)$ of (20) over the sink flows $p_{i}(x), i=1 \ldots n$, we have

$$
\max _{p_{i}(x) \leq \rho\left(l_{i}, x\right)} \int_{\Omega} u_{i} p_{i} d x=\int_{\Omega} u_{i}(x) \rho\left(l_{i}, x\right) d x, \quad u_{i}(x) \geq 0, i=1, \ldots, n .
$$

For the maximization over the spatial flow functions $q_{i}(x), i=1, \ldots, n$, it is well-known [31] that

$$
\max _{\left|q_{i}(x)\right| \leq C_{i}(x)} \int_{\Omega} u_{i} \operatorname{div} q_{i} d x=\int_{\Omega} C_{i}(x)\left|\nabla u_{i}\right| d x .
$$

Furthermore, observe the source flow function $p_{s}(x)$ is unconstrained, the maximization of (20) over $p_{s}$ simply leads to

$$
1-\sum_{i=1}^{n} u_{i}(x)=0, \quad \forall x \in \Omega .
$$

By the results of (28), (26) and (27), it is easy to conclude that the maximization of the primal-dual model (20) over flow functions $p_{s}, p$ and $q$ gives its equivalent dual model (22), hence we have

Proposition 1. The continuous max-flow model (16), the primal-dual model (19) and the dual model (22) are equivalent to each other.

In this work, we focus on the case when $C_{i}(x)=\alpha, \forall x \in \Omega$ and $i=1, \ldots, n$. Obviously, we have

Proposition 2. When $C_{i}(x)=\alpha, \forall x \in \Omega$ and $i=1 \ldots n$, the dual model (22) equals the continuous min-cut model (5). 


\subsection{Variational Perspective of Flows and Cuts}

Through the above analytical results, we can also give a variational perspective of flows and cuts, which recovers conceptions and terminologies used in the graph setting.

Consider the maximization problem (23), for any fixed $q$, let some optimal $p^{*}$ maximize $q \cdot p$ over $p \leq C$. By means of variations, if such $p^{*}<C$ strictly, its variation directly leads to $q=0$ since the variation $\delta p$ can be both negative and positive. On the other hand, for $p^{*}=C$, its variation under the constraint $p \leq C$ gives $\delta p<0$, then we must have $q>0$. In terms of graph-cut, $p^{*}<C$ means $p$ does not reach its maximum $C$, i.e. 'unsaturated'; then it leads to $q=0$ which means the so-called 'cut'.

In the same manner, for the maximization of $p_{i}(x), i=1 \ldots n$, it is easy to see that when the flow $p_{i}(x)<\rho\left(l_{i}, x\right)$ at $x \in \Omega$, i.e. 'unsaturated', we must have $u_{i}(x)=0$, i.e. $u_{i}(x) p_{i}(x)=0$, which means that at the position $x$, the flow $p_{i}(x)$ has no contribution to the energy function and the flow $p_{i}(x)$, from $x \in \Omega_{i}$ to the sink $t$, can be 'cut' off from the energy function of (19). On the other hand, in view of (8), the indicator function $u_{i}(x)=0$ definitely means the position $x$ is not labeled as $l_{i}$.

\section{Multiplier-Based Max-Flow Algorithm}

Observe that the energy function of the primal-dual model (19) just gives the Lagrangian function of (16) where $u_{i}(x), i=1 \ldots n$, are the corresponding multiplier functions. We introduce our multiplier-based max-flow algorithm, which is based on the augmented lagrangian method [23]. We define the augmented Lagrangian function

$L_{c}\left(p_{s}, p, q, u\right)=\int_{\Omega} p_{s} d x+\sum_{i=1}^{n}\left\langle u_{i}, \operatorname{div} q_{i}-p_{s}+p_{i}\right\rangle-\frac{c}{2} \sum_{i=1}^{n}\left\|\operatorname{div} q_{i}-p_{s}+p_{i}\right\|^{2}$

where $c>0$. Each iteration of the algorithm can then be generalized as follows:

- Optimize spatial flows $q_{i}, i=1 \ldots n$, by fixing other variables:

$$
q_{i}^{k+1}:=\arg \max _{\left\|q_{i}\right\|_{\infty} \leq \alpha}-\frac{c}{2}\left\|\operatorname{div} q_{i}+p_{i}^{k}-p_{s}^{k}-u_{i}^{k} / c\right\|^{2},
$$

which can be solved by Chambolle's projection algorithm [32].

- Optimize sink flows $p_{i}, i=1 \ldots n$, by fixing other variables

$$
p_{i}^{k+1}:=\arg \max _{p_{i}(x) \leq \rho\left(\ell_{i}, x\right)}-\frac{c}{2}\left\|p_{i}+\operatorname{div} q_{i}^{k+1}-p_{s}^{k}-u_{i}^{k} / c\right\|^{2},
$$

which can be computed at each $x \in \Omega$ in a closed form. 
- Optimize the source flow $p_{s}$ and update multipliers $u_{i}, i=1 \ldots n$

$$
\begin{aligned}
p_{s}^{k+1}:=\arg \max _{p_{s}} \int_{\Omega} p_{s} d x-\frac{c}{2} \sum_{i=1}^{n}\left\|p_{s}-\left(p_{i}^{k+1}+\operatorname{div} q_{i}^{k+1}\right)+u_{i}^{k} / c\right\|^{2}, \\
u_{i}^{k+1}=u_{i}^{k}-c\left(\operatorname{div} q_{i}^{k+1}-p_{s}^{k+1}+p_{i}^{k+1}\right) .
\end{aligned}
$$

Both can be obtained in a closed form.

Consider the above numerical steps, it is easy to see that the two flows $q_{i}$ and $p_{i}, i=1 \ldots n$, computed by (29) and (30) can be handled independently for each label $i$. Hence, (29) and (30) can be implemented in a parallel way. Once such two steps are finished, the source flow $p_{s}(x)$ and the labeling functions $u_{i}(x), i=1 \ldots n$, are updated. Obviously, such parallelism naturally originates the configuration shown in Fig. 1.

\section{Experiments}

In this section, we show some experiments to validate the proposed max-flow model and its resulted algorithm. The quality of the relaxation (5) has been evaluated extensively in $[18,22,21]$ where it has been shown to be competitive to several state of the art methods from discrete optimization like alpha expansion and alpha beta-swap [2] for approximately minimizing the Pott's energy. In addition the variational model comes with the important advantage of rotational invariance, which means that metrication errors are avoided. We will therefore not elaborate too much on the quality of the solutions in this paper. Examples are given in Figure (2), where we have used the Mumford-Shah data term $\rho\left(\ell_{i}, x\right)=\left|I(x)-\ell_{i}\right|^{2}, i=1, \ldots, n$. As we see, equally good solutions as alpha expansion are produced, but without the metrication artifacts.

In contrast to the minimization approach of Zach et. al. [18], the proposed algorithm can be proved to converge by classical optimization theories. The Douglas-Rachford splitting approach given in [22] can also be proved to converge (in the discrete setting), but we experienced that our approach was more efficient than both these approaches. The inner problem has the same complexity for all approaches, since it is dominated by the process of iteratively solve a tv minimization problem. However, in contrast to $[18,22]$ our approach avoids iterative projections to the convex set $S$ and consequently require much less outer iterations. Convergence is reached for a wide range of the outer "step size" $c$. To measure converge, we first find a good estimate of the ground truth energy $E^{*}$ by solving the problem with 10000 outer iterations. The energy precision at iteration $k$ is then measured by

$$
\epsilon=\frac{E^{k}-E^{*}}{E^{*}} .
$$

For the three images (see Fig. 2), different precision $\epsilon$ are taken and the total number of iterations to reach convergence is evaluated, see Tab 1: clearly, our method is about 4 times faster than the Douglas-Rachford-splitting [22], the approach in [18] is even slower and failed to reach such a low precision. 

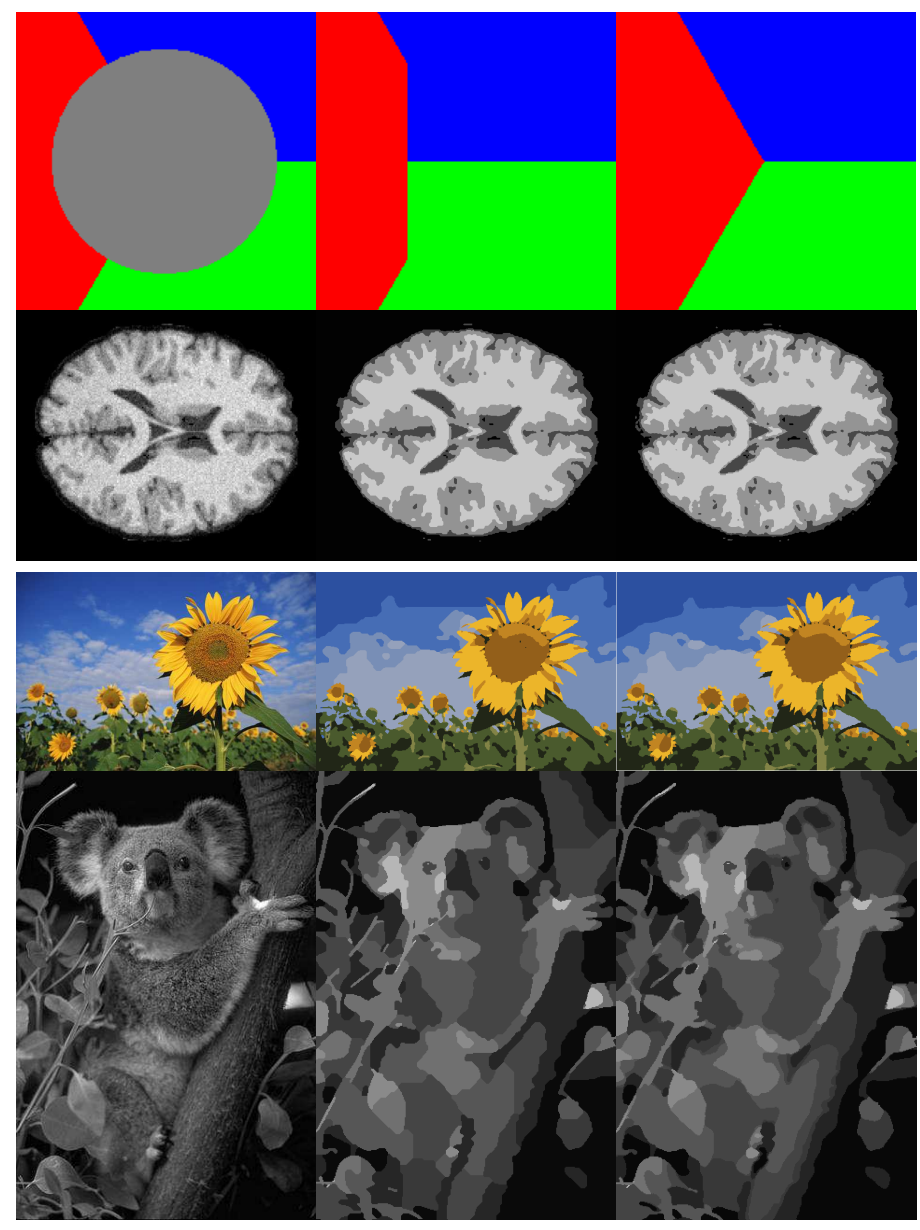

Fig. 2. Each row (from left to right): the input image, result by Alpha expansion with 8 neighbors, result by the proposed max-flow approach. For the inpainting experiment (1st row), $\alpha=0.03$ and $n=3$; for the experiment at 2 nd row, $\alpha=0.047$ and $n=10$; for the experiment at 3rd row, $\alpha=0.02$ and $n=8$; for the experiment at 4 th row, $\alpha=0.04$ and $n=4$.

\section{Conclusions}

In this paper, we introduce and study the novel continuous max-flow model which is dual to the continuous min-cut problem, i.e. the convex relaxed Potts model. We also propose a variational perspective of flows and cuts in the continuous configuration, which recovers and well explains connections of flows and cuts. Moreover, in comparison to previous efforts which are trying to compute the optimal labeling functions in a direct way, we propose the new multiplier-based max-flow algorithm. Main advantages of such max-flow algorithm are: it avoids 


\begin{tabular}{|l|c|c|c|}
\hline & Brain $\epsilon \leq 10^{-5}$ & Flower $\epsilon \leq 10^{-4}$ & Bear $\epsilon \leq 10^{-4}$ \\
\hline Zach et al [18] & \multicolumn{3}{|c|}{ fail to reach such a precision } \\
\hline Lellmann et al $[22]$ & 421 iter. & 580 iter. & 535 iter. \\
\hline Proposed algorithm & 88 iter. & 147 iter. & 133 iter. \\
\hline
\end{tabular}

Table 1. Comparisons between algorithms: Zach et al [18], Lellmann [22] and the proposed max-flow algorithm: for the three images (see Fig. 2), different precision $\epsilon$ are taken and the total number of iterations to reach convergence is evaluated.

extra computation load to explore the simplex constraint, each flow is adjusted in a simple way and its numerical scheme contains a natural parallel framework, which can be easily accelarated. Numerical experiments show it outperforms state of art approaches in terms of quality and efficiency.

Acknowledgements: This research has been supported by Natural Sciences and Engineering Research Council of Canada (NSERC) Accelerator Grant R3584A04, the Norwegian Research Council (eVita project 166075 and MOE (Ministry of Education) Tier II project T207N2202).

\section{References}

1. Paragios, N., Chen, Y., Faugeras, O.: Handbook of Mathematical Models in Computer Vision. Springer-Verlag New York, Inc., Secaucus, NJ, USA (2005)

2. Boykov, Y., Veksler, O., Zabih, R.: Fast approximate energy minimization via graph cuts. IEEE Transactions on PAMI 23 (2001) 1222 - 1239

3. Nikolova, M., Esedoglu, S., Chan, T.F.: Algorithms for finding global minimizers of image segmentation and denoising models. SIAM J. App. Math. 66 (2006) 1632-1648

4. Kolmogorov, V., Zabih, R.: Multi-camera scene reconstruction via graph cuts. In: European Conference on Computer Vision. (2002) 82-96

5. Li, S.Z.: Markov random field modeling in image analysis. Springer-Verlag New York, Inc., Secaucus, NJ, USA (2001)

6. Greig, D.M., Porteous, B.T., Seheult, A.H.: Exact maximum a posteriori estimation for binary images. J. Royal Stat. Soc., Series B (1989) 271-279

7. Kolmogorov, V., Zabih, R.: What energy functions can be minimized via graph cuts. IEEE Transactions on PAMI 26 (2004) 65-81

8. Komodakis, N., Tziritas, G.: Approximate labeling via graph-cuts based on linear programming. In: Pattern Analysis and Machine Intelligence. (2007) 1436-1453

9. Wainwright, M., Jaakkola, T., Willsky, A.: Map estimation via agreement on (hyper)trees: Message-passing and linear programming approaches. IEEE Transactions on Information Theory 51 (2002) 3697-3717

10. Boykov, Y., Kolmogorov, V.: Computing geodesics and minimal surfaces via graph cuts. In: ICCV 2003. (2003) 26

11. Kolmogorov, V., Boykov, Y.: What metrics can be approximated by geo-cuts, or global optimization of length/area and flux. In: In ICCV. (2005) 564-571

12. Kohli, P., Kumar, M.P., Torr, P.H.: $p^{3}$ and beyond: Move making algorithms for solving higher order functions. IEEE Transactions on PAMI 31 (2009) 1645-1656 
13. Osher, S., Sethian, J.: Fronts propagating with curvature dependent speed: algorithms based on hamilton-jacobi formulations. J. Comput. Phys. 79 (1988) 12-49

14. Chan, T., Vese, L.: Active contours without edges. IEEE Image Proc., 10, pp. 266-277 (2001)

15. Vese, L.A., Chan, T.F.: A new multiphase level set framework for image segmentation via the mumford and shah model. IJCV 50 (2002) 271-293

16. Lie, J., Lysaker, M., Tai, X.: A binary level set model and some applications to Mumford-Shah image segmentation. IEEE Img. Proc. 15 (2006) 1171-1181

17. Lie, J., Lysaker, M., Tai, X.C.: A variant of the level set method and applications to image segmentation. Math. Comp. 75 (2006) 1155-1174

18. Zach, C., Gallup, D., Frahm, J.M., Niethammer, M.: Fast global labeling for realtime stereo using multiple plane sweeps. In: VMV 2008. (2008)

19. Pock, T., Schoenemann, T., Graber, G., Bischof, H., Cremers, D.: A convex formulation of continuous multi-label problems. In: ECCV 2008. (2008)

20. Chambolle, A., Cremers, D., Pock, T.: A convex approach for computing minimal partitions. Technical Report TR-2008-05, University of Bonn (2008)

21. Bae, E., Yuan, J., Tai, X.: Convex relaxation for multipartitioning problems using a dual approach. Technical report CAM09-75, UCLA, CAM (2009)

22. Lellmann, J., Kappes, J., Yuan, J., Becker, F., Schnörr, C.: Convex multi-class image labeling by simplex-constrained total variation. Technical report, HCI, IWR, Uni. Heidelberg (2008)

23. Bertsekas, D.P.: Nonlinear Programming. Athena Scientific (1999)

24. Cormen, T.H., Leiserson, C.E., Rivest, R.L., Stein, C.: Introduction to Algorithms. Second edn. MIT Press, Cambridge, MA (2001)

25. Potts., R.B.: Some generalized order-disorder transformations. In Proceedings of the Cambridge Philosophical Society, Vol. 48 (1952) 106-109

26. Pock, T., Chambolle, A., Bischof, H., Cremers, D.: A convex relaxation approach for computing minimal partitions. In: CVPR, Miami, Florida (2009)

27. Yuan, J., Bae, E., Tai, X.: A study on continuous max-flow and min-cut approaches. In: CVPR, USA, San Francisco (2010)

28. Bresson, X., Esedoglu, S., Vandergheynst, P., Thiran, J., Osher, S.: Fast global minimization of the active contour/snake model. Journal of Mathematical Imaging and Vision 28 (2007) 151-167

29. Ekeland, I., Téman, R.: Convex analysis and variational problems. Society for Industrial and Applied Mathematics, Philadelphia, PA, USA (1999)

30. Fan, K.: Minimax theorems. Proc. Nat. Acad. Sci. U. S. A. 39 (1953) 42-47

31. Giusti, E.: Minimal surfaces and functions of bounded variation. Australian National University, Canberra (1977)

32. Chambolle, A.: An algorithm for total variation minimization and applications. Journal of Mathematical Imaging and Vision 20 (2004) 89-97 